\title{
An efficient one-pot synthesis of novel pyrazolophthalazinyl spirooxindoles
}

\author{
GNANAMANI SHANTHI and PARAMASIVAN T PERUMAL* \\ Organic Chemistry Division, Central Leather Research Institute, Chennai 600020 \\ e-mail: ptperumal@gmail.com
}

MS received 25 August 2008; revised 31 October 2009; accepted 26 November 2009

\begin{abstract}
A simple and efficient method for the synthesis of novel pyrazolophthalazinyl spirooxindoles by L-proline catalysed one-pot three-component reaction is described.
\end{abstract}

Keywords. L-proline; pyrazolophthalazinyl spirooxindoles; three-component.

\section{Introduction}

Multicomponent reactions (MCRs) are special types of synthetically useful organic reactions in which three or more different starting materials react to a final product in a one-pot procedure. MCRs are powerful tools in the modern drug discovery process and allow the fast, automated, and high-throughput generation of organic compounds. ${ }^{1}$ In the past decade, there have been tremendous developments in three- and four-component reactions and efforts are still being made to find and develop new MCRs. ${ }^{2}$ The indole template is generally recognized as an important structure in medicinal chemistry, and in particular, oxindoles that incorporate a quaternary stereogenic centre at $\mathrm{C} 3$ are attractive targets in organic synthesis because of their significant biological activities. ${ }^{3}$ The spirooxindole system is the core structure of many pharmacological agents and natural alkaloids. For example, spirotryprostatin A, a natural alkaloid isolated from the fermentation broth of Aspergillus fumigatus, has been identified as a novel inhibitor of microtubule assembly, ${ }^{4}$ and pteropodine and isopteropodine have been shown to modulate the function of muscarinic serotonin receptors (figure 1). ${ }^{5}$ In addition to the naturally occurring pyrrolidino-spiro-3'-oxindoles, synthetic pyrrolino- and piperidino-spiro- 3 '-oxindoles have been shown to exhibit local anaesthetic properties. The unique structural array and the highly pronounced pharmacological activity displayed by the

\footnotetext{
*For correspondence
}

class of spirooxindoles have made them attractive synthetic targets (figure 1).

\section{Experimental}

\subsection{Materials and methods}

Malononitrile, isatin, $\mathrm{NH}_{2} \mathrm{SO}_{3} \mathrm{H}, \mathrm{SnCl}_{2} \cdot 2 \mathrm{H}_{2} \mathrm{O}, \mathrm{K}_{2} \mathrm{CO}_{3}$ and basic alumina were obtained from S.D. Fine Chemicals Indium(III) chloride, CAN, L-proline and phthalhydrazide were purchased from Aldrich. All melting points were uncorrected. IR spectra were recorded on a Perkin Elmer FT-IR spectrophotometer. ${ }^{1} \mathrm{H}$ and ${ }^{13} \mathrm{C}$ NMR spectra were recorded in DMSO- $d_{6}$ using TMS as an internal standard on a JEOL spectrometer at $500 \mathrm{MHz}$ and $125 \mathrm{MHz}$ and Bruker spectrometer at $300 \mathrm{MHz}$ and $75 \mathrm{MHz}$ respectively. Mass spectra were recorded on a JEOL

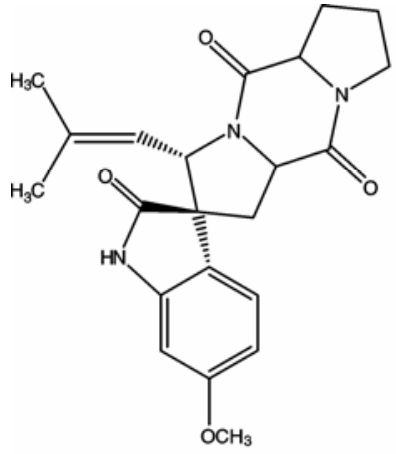

Spirotryprostatin A

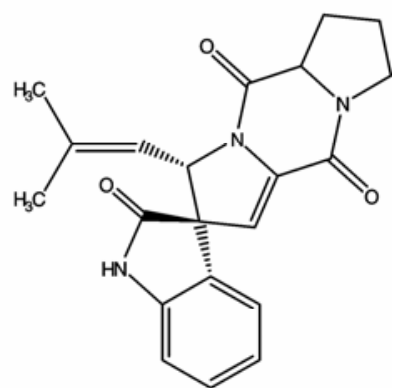

Spirotryprostatin B
Figure 1. Representatives of spirooxindole-containing compounds. 
Table 1. Synthesis of pyrazolophthalazinyl spiro-3'-oxindoles $\mathbf{4 a - j}$.

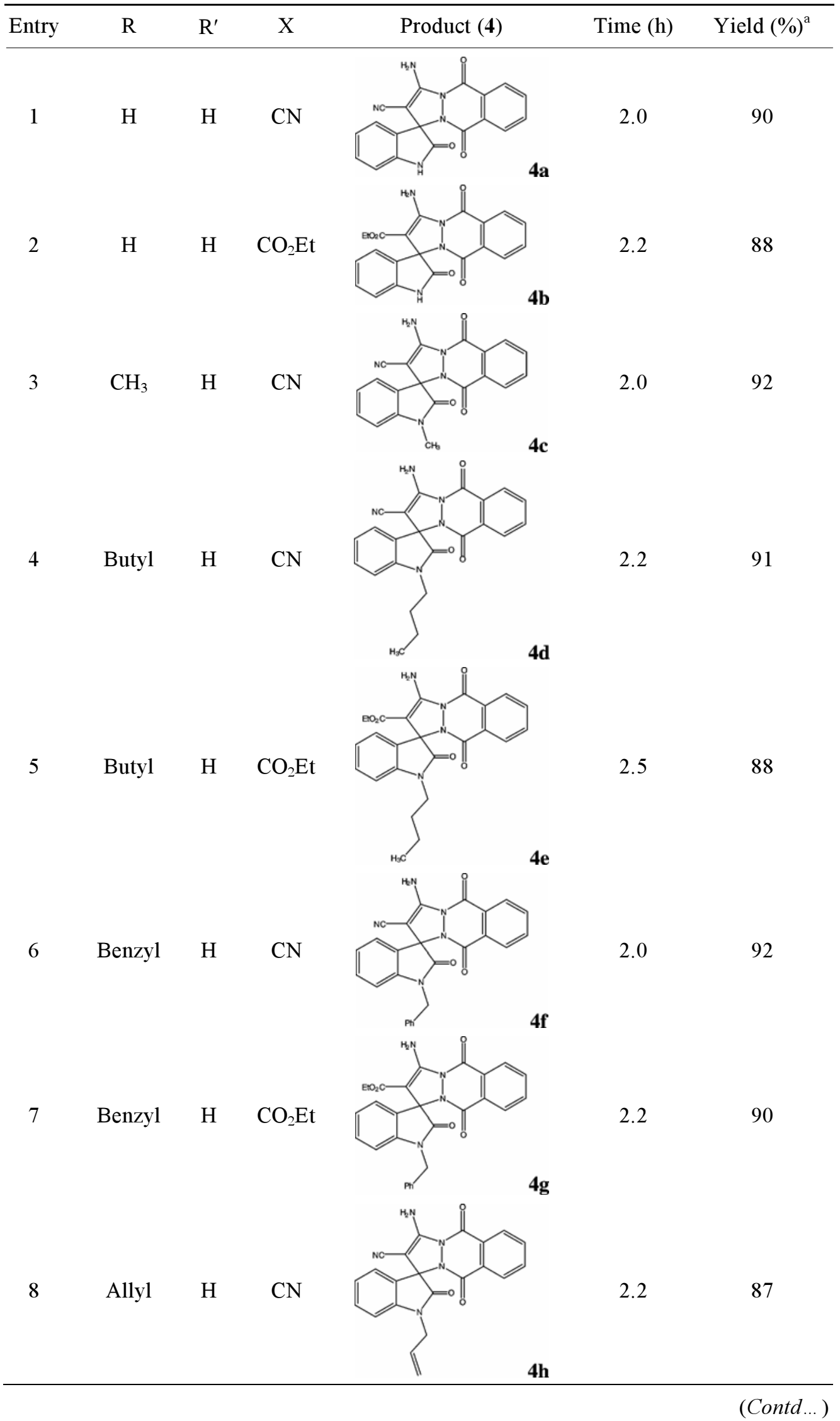


Table 1. (Contd...)

\begin{tabular}{|c|c|c|c|c|c|c|}
\hline Entry & $\mathrm{R}$ & $\mathrm{R}^{\prime}$ & $\mathrm{X}$ & Product (4) & Time (h) & Yield $(\%)^{\mathrm{a}}$ \\
\hline 9 & Allyl & $\mathrm{H}$ & $\mathrm{CO}_{2} \mathrm{Et}$ & & 2.5 & 86 \\
\hline 10 & $\mathrm{H}$ & $\mathrm{NO}_{2}$ & $\mathrm{CN}$ & & 2.0 & 90 \\
\hline
\end{tabular}

${ }^{\mathrm{a}}$ Isolated yield

DX 303 HF spectrometer. Elemental analyses were recorded using a Thermo Finnigan FLASH EA 1112 CHN analyser. Analytical TLC was performed on pre-coated plastic sheets of silica gel G/UV-254 of $0.2 \mathrm{~mm}$ thickness (Macherey-Nagel, Germany).

2.2 General procedure for the synthesis of pyrazolophthalazinyl spirooxindoles $(\mathbf{4 a - j})$

L-proline (20 mol\%) was added to a stirred mixture of isatin (1 mmol), malononitrile $(1 \mathrm{mmol})$ and phthalhydrazide $(1 \mathrm{mmol})$ in ethanol $(5 \mathrm{~mL})$, and refluxed for the appropriate time (table 1). After complete conversion as indicated by TLC, water was added and the product was extracted with ethyl acetate $(2 \times 15 \mathrm{~mL})$. The combined extracts were dried over anhydrous $\mathrm{Na}_{2} \mathrm{SO}_{4}$ and concentrated in vacuo. The resulting product was recrystallized from ethanol to afford pure product $(\mathbf{4} \mathbf{a}-\mathbf{j})$.

2.2a 3-Amino-5,10-dioxo spiro[(3 H)-indol-3', 15,10-dihydro-1(H)-pyrazolo(1,2-b)phthalazin]-(1 H)2'-one-2-carbonitrile (4a) (table 1, entry 1): yellow solid. m.p.: 269-270 ${ }^{\circ} \mathrm{C}$. $v_{\max }(\mathrm{KBr}): 3439,3350$, 2209, 1756, 1678, 1653, 1465, 1364, 1257, $1163 \mathrm{~cm}^{-1}$. ${ }^{1} \mathrm{H}$ NMR (DMSO- $d_{6}, 500 \mathrm{MHz}$ ): $\delta 6.90$ $(d, 1 \mathrm{H}, J=7.65 \mathrm{~Hz}), 6.97(t, 1 \mathrm{H}, J=7.65 \mathrm{~Hz}), 7.27$ $(t, 1 \mathrm{H}, J=7.6 \mathrm{~Hz}), 7.44(d, 1 \mathrm{H}, J=7.65 \mathrm{~Hz}), 7.96$ $(m, 3 \mathrm{H}), 8.25(d, 1 \mathrm{H}, J=7.65 \mathrm{~Hz}), 8.32(b r s, 2 \mathrm{H}$, $\mathrm{NH}_{2}, \mathrm{D}_{2} \mathrm{O}$ exchangeable), $10.92\left(s, 1 \mathrm{H}, \mathrm{NH}, \mathrm{D}_{2} \mathrm{O}\right.$ exchangeable). ${ }^{13} \mathrm{C}$ NMR (DMSO- $\left.d_{6}, 125 \mathrm{MHz}\right): \delta$ $60 \cdot 6,70 \cdot 3,110 \cdot 9,114 \cdot 9,123 \cdot 1,125 \cdot 2,125 \cdot 9,127 \cdot 5$, $128 \cdot 1,128 \cdot 3,129 \cdot 2,131 \cdot 0,134.9,135.6,142 \cdot 7$, $152 \cdot 2,153 \cdot 1,156 \cdot 9,173 \cdot 0$. MS $(m / z): 357\left(\mathbf{M}^{+}\right)$.
Anal. Calcd. For $\mathrm{C}_{19} \mathrm{H}_{11} \mathrm{~N}_{5} \mathrm{O}_{3}: \mathrm{C}, 63 \cdot 87 ; \mathrm{H}, 3 \cdot 10 ; \mathrm{N}$, $19.60 \%$. Found: C, 63.83; H, 3.06; N, 19.55\%.

2.2b Ethyl 3-amino-5,10-dioxo spiro[(3 $H)$-indol3',1-5,10-dihydro-1(H)-pyrazolo(1,2-b) phthalazin](1 H)-2'-one-2-carboxylate (4b) (table 1, entry 2): yellow solid. m.p.: 284-286 ${ }^{\circ} \mathrm{C}$. $v_{\max }(\mathrm{KBr}): 3438$, $3328,1742,1701,1665,1527,1294,1140 \mathrm{~cm}^{-1} \cdot{ }^{1} \mathrm{H}$ NMR (DMSO- $\left.d_{6}, 500 \mathrm{MHz}\right): \delta 0.84(t, 3 \mathrm{H}, J=$ $7.65 \mathrm{~Hz}), 3.81(m, 2 \mathrm{H}), 6.80(d, 1 \mathrm{H}, J=7.65 \mathrm{~Hz})$, $6.84(t, 1 \mathrm{H}, J=6.85 \mathrm{~Hz}), 7.18(t, 1 \mathrm{H}, J=7.65 \mathrm{~Hz})$, $7.27(d, 1 \mathrm{H}, J=7.65 \mathrm{~Hz}), 7.52\left(\right.$ br $s, 2 \mathrm{H}, \mathrm{NH}_{2}, \mathrm{D}_{2} \mathrm{O}$ exchangeable $), 7.94(m, 3 \mathrm{H}), 8.25(d, 1 \mathrm{H}, J=$ $9.15 \mathrm{~Hz}), 10.73\left(s, 1 \mathrm{H}, \mathrm{NH}, \mathrm{D}_{2} \mathrm{O}\right.$ exchangeable $) .{ }^{13} \mathrm{C}$ NMR (DMSO- $\left.d_{6}, 125 \mathrm{MHz}\right): \delta 14.2,59 \cdot 3,70.7$, $81 \cdot 3,109 \cdot 9,122 \cdot 2,124 \cdot 3,127 \cdot 3,127 \cdot 5,128 \cdot 0$, $128 \cdot 6,129 \cdot 1,130 \cdot 0,134 \cdot 7,135 \cdot 5,143 \cdot 9,151 \cdot 2$, 152.6, 157.0, 163.7, 173.5. MS $(\mathrm{m} / \mathrm{z}): 404\left(\mathbf{M}^{+}\right)$. Anal. Calcd. For $\mathrm{C}_{21} \mathrm{H}_{16} \mathrm{~N}_{4} \mathrm{O}_{5}$ : C, 62.37; H, 3.99; N, $13.85 \%$. Found: C, 62.28; H, 3.94; N, 13.78\%.

2.2c 3-Amino-1'-methyl-5,10-dioxo spiro[(3 $\mathrm{H})$ indol-3', 1-5, 10-dihydro-1(H)-pyrazolo(1,2-b)phthalazin]-(1 H)-2'-one-2-carbonitrile (4c) (table 1, entry $3)$ : pale yellow solid. m.p.: $282-284^{\circ} \mathrm{C} . v_{\max }(\mathrm{KBr})$ : 3453, 3327, 2197, 1728, 1664, 1611, 1472, 1371, $698 \mathrm{~cm}^{-1} .{ }^{1} \mathrm{H}$ NMR (DMSO- $\left.d_{6}, 500 \mathrm{MHz}\right): \delta 3 \cdot 22(s$, $3 \mathrm{H}), \quad 7.05(t, 1 \mathrm{H}, J=7.65 \mathrm{~Hz}), 7.11 \quad(d, 1 \mathrm{H}$, $J=7.65 \mathrm{~Hz}), 7.37(t, 1 \mathrm{H}, J=8.4 \mathrm{~Hz}), 7.51(d, 1 \mathrm{H}$, $J=6.9 \mathrm{~Hz}), 7.95(m, 3 \mathrm{H}), 8.26(d, 1 \mathrm{H}, J=8.4 \mathrm{~Hz})$, 8.37 (br s, 2H, $\mathrm{NH}_{2}, \mathrm{D}_{2} \mathrm{O}$ exchangeable). ${ }^{13} \mathrm{C} \mathrm{NMR}$ $\left(\mathrm{DMSO}-d_{6}, 125 \mathrm{MHz}\right): \delta 27.3,60.0,69.9,109.7$, $114.9,123 \cdot 8,124.9,125 \cdot 3,127 \cdot 5,128 \cdot 1,128 \cdot 3,129 \cdot 2$, $131 \cdot 2,135 \cdot 0,135 \cdot 6,144 \cdot 2,152 \cdot 4,153 \cdot 1,156 \cdot 8,171 \cdot 6$. 
MS $(\mathrm{m} / z)$ : $371\left(\mathbf{M}^{+}\right)$. Anal. Calcd. for $\mathrm{C}_{20} \mathrm{H}_{13} \mathrm{~N}_{5} \mathrm{O}_{3}$ : C, 64.69; H, 3.53; N, 18.86\%. Found: C, 64.63; H, $3 \cdot 48 ; \mathrm{N}, 18 \cdot 81 \%$.

2.2d 3-Amino-1 '-butyl-5, 10-dioxo spiro[(3 'H)-indol3', 1-5, 10-dihydro-1(H)-pyrazolo(1,2-b)phthalazin](1 H)-2'-one-2-carbonitrile (4d) (table 1, entry 4): pale yellow solid. m.p.: $222-223^{\circ} \mathrm{C} . \quad v_{\max }(\mathrm{KBr})$ : $3382,3260,2937,2196,1668,1610,1562,1467$, $1432,1374,1257,1146 \mathrm{~cm}^{-1} .{ }^{1} \mathrm{H}$ NMR (DMSO- $d_{6}$, $500 \mathrm{MHz}): \delta 0.87(t, 3 \mathrm{H}, J=6.9 \mathrm{~Hz}), 1.33(m, 2 \mathrm{H})$, $1.59(m, 2 \mathrm{H}), 3.70 \quad(m, 2 \mathrm{H}), 7.03(t, 1 \mathrm{H}, J=$ $7.65 \mathrm{~Hz}), 7.14(d, 1 \mathrm{H}, J=7.65 \mathrm{~Hz}), 7.35(t, 1 \mathrm{H}, J=$ $7.6 \mathrm{~Hz}), 7.51(d, 1 \mathrm{H}, J=6.85 \mathrm{~Hz}), 7.94(t, 2 \mathrm{H}$, $J=7.6 \mathrm{~Hz}), 8.00(d, 1 \mathrm{H}, J=8.45 \mathrm{~Hz}), 8.26(d, 1 \mathrm{H}$, $J=7.6 \mathrm{~Hz}), 8.35\left(b r s, 2 \mathrm{H}, \mathrm{NH}_{2}, \mathrm{D}_{2} \mathrm{O}\right.$ exchangeable). ${ }^{13} \mathrm{C}$ NMR (DMSO- $d_{6}, 125 \mathrm{MHz}$ ): $\delta 14 \cdot 2,19 \cdot 8$, $29 \cdot 5,60 \cdot 4,70 \cdot 0,109 \cdot 9,114 \cdot 8,123 \cdot 5,125 \cdot 0,125 \cdot 4$, $127 \cdot 5,128 \cdot 1,128 \cdot 3,129.2,131 \cdot 1,134.9,135 \cdot 6$, $143.5,152.3,153 \cdot 1,156 \cdot 9,171.4$. MS $(\mathrm{m} / z): 413$ $\left(\mathrm{M}^{+}\right)$. Anal. Calcd. for $\mathrm{C}_{23} \mathrm{H}_{19} \mathrm{~N}_{5} \mathrm{O}_{3}$ : C, 66.82; H, $4.63 ; \mathrm{N}, 16.94 \%$. Found: C, 66.93; H, 4.58; N, $16.88 \%$.

2.2e Ethyl 3-amino-1'-butyl-5,10-dioxo spiro[(3 $\mathrm{H})$ indol-3,'1-5,10-dihydro-1(H)-pyrazolo(1,2-b) phthalazin]-(1 H)-2'-one-2-carboxylate (4e) (table 1, entry 5): pale yellow solid. m.p.: $258-260^{\circ} \mathrm{C}$. $v_{\max }(\mathrm{KBr})$ : $3427,3319,2933,1731,1704,1674,1610,1527$, $1468,1428,1378,1298,1263,1141,757,699 \mathrm{~cm}^{-1}$. ${ }^{1} \mathrm{H}$ NMR (DMSO- $\left.d_{6}, 500 \mathrm{MHz}\right): \delta 0.77(m, 3 \mathrm{H})$, $0.89(t, 3 \mathrm{H}, J=6.85 \mathrm{~Hz}), 1.37(m, 2 \mathrm{H}), 1.60(\mathrm{~m}$, $2 \mathrm{H}), 3.67(t, 2 \mathrm{H}, J=7.65 \mathrm{~Hz}), 3.81(m, 2 \mathrm{H}), 6.91(t$, $1 \mathrm{H}, J=7.6 \mathrm{~Hz}), 7.02(d, 1 \mathrm{H}, J=8.4 \mathrm{~Hz}), 7.26(t$, $1 \mathrm{H}, J=7.65 \mathrm{~Hz}), 7.33(d, 1 \mathrm{H}, J=7.65 \mathrm{~Hz}), 7.95(m$, $3 \mathrm{H}), 7.62$ (br s, 2H, $\mathrm{NH}_{2}, \mathrm{D}_{2} \mathrm{O}$ exchangeable), 8.26 (d) $1 \mathrm{H}, \quad J=6.85 \mathrm{~Hz}) .{ }^{13} \mathrm{C}$ NMR (DMSO- $d_{6}$, $125 \mathrm{MHz}$ ): $\delta 14 \cdot 1,14 \cdot 3,20 \cdot 0,29 \cdot 7,59 \cdot 2,70 \cdot 1,80 \cdot 2$, $108 \cdot 8,122 \cdot 7,124 \cdot 1,126 \cdot 8,127 \cdot 6,128 \cdot 0,128 \cdot 6$, $129 \cdot 1,130.2,134 \cdot 8,135.5,144 \cdot 7,151.4,152.7$, 157.2, 163.6, 171.8. MS $(\mathrm{m} / \mathrm{z}): 460\left(\mathbf{M}^{+}\right)$. Anal. Calcd. for $\mathrm{C}_{25} \mathrm{H}_{24} \mathrm{~N}_{4} \mathrm{O}_{5}: \mathrm{C}, 65.21 ; \mathrm{H}, 5.25 ; \mathrm{N}$, $12 \cdot 17 \%$. Found: C, $65 \cdot 27$; H, 5.20; N, 12.12\%.

$2.2 \mathrm{f}$ 3-Amino-1'-benzyl-5,10-dioxo spiro[ $[(3 \mathrm{H})$ indol-3',1-5, 10-dihydro-1(H)-pyrazolo(1,2-b)phthalazin]-(1 H)-2'-one-2-carbonitrile (4f) (table 1, entry 6): pale yellow solid. m.p.: $266^{\circ} \mathrm{C} . v_{\max }(\mathrm{KBr}): 3373$, $3258,2197,1666,1611,1468,1371,1163,698 \mathrm{~cm}^{-1}$. ${ }^{1} \mathrm{H}$ NMR (DMSO- $\left.d_{6}, 500 \mathrm{MHz}\right): \delta 4.96(\mathrm{ABq}, 2 \mathrm{H}$, $J=16.05 \mathrm{~Hz}), 6.87(d, 1 \mathrm{H}, J=8.4 \mathrm{~Hz}), 7.03 \quad(t$,
$1 \mathrm{H}, J=6.85 \mathrm{~Hz}), 7.26(d, 2 \mathrm{H}, J=6.9 \mathrm{~Hz}), 7.30$ $(t, 2 \mathrm{H}, J=7.65 \mathrm{~Hz}), 7.42(d, 2 \mathrm{H}, J=7.6 \mathrm{~Hz}), 7.56$ $(d, 1 \mathrm{H}, J=7.65 \mathrm{~Hz}), 7.97(m, 2 \mathrm{H}), 8.05(d, 1 \mathrm{H}$, $J=6.85 \mathrm{~Hz}), 8.28(d, 1 \mathrm{H}, J=9.15 \mathrm{~Hz}), 8.41(b r s$, $2 \mathrm{H}, \mathrm{NH}_{2}, \mathrm{D}_{2} \mathrm{O}$ exchangeable). ${ }^{13} \mathrm{C}$ NMR (DMSO- $d_{6}$, $125 \mathrm{MHz}): \delta 44 \cdot 0,60 \cdot 3,70 \cdot 0,110 \cdot 3,115 \cdot 0,123 \cdot 9$, $125 \cdot 1,125 \cdot 4,127 \cdot 5,127 \cdot 6,128 \cdot 0,128 \cdot 2,129 \cdot 1$, $129 \cdot 3,131 \cdot 0,135 \cdot 0,135 \cdot 6,136 \cdot 0,143 \cdot 1,152 \cdot 4$, 153.2, 156.9, 171.8. MS $(\mathrm{m} / \mathrm{z}): 447\left(\mathbf{M}^{+}\right)$. Anal. Calcd. for $\mathrm{C}_{26} \mathrm{H}_{17} \mathrm{~N}_{5} \mathrm{O}_{3}: \mathrm{C}, 69.79 ; \mathrm{H}, 3.83 ; \mathrm{N}$, $15 \cdot 65 \%$. Found: C, 69.72; H, 3.78; N, 15.60\%.

$2.2 \mathrm{~g}$ Ethyl 3-amino-1'-benzyl-5, 10-dioxo spiro [(3 'H)-indol-3', 1-5, 10-dihydro-1 (H)-pyrazolo (1,2b)phthalazin]-(1 H)-2'-one-2-carboxylate (4g) (table 1, entry 7): pale yellow solid. m.p.: $290-291^{\circ} \mathrm{C} . v_{\max }$ $(\mathrm{KBr}): 3426,3316,1702,1697,1528,1428,1383$, 1298, 1263, 1143, $700 \mathrm{~cm}^{-1}$. ${ }^{1} \mathrm{H}$ NMR (DMSO- $d_{6}$, $500 \mathrm{MHz}): \delta 0.65(m, 3 \mathrm{H}), 3.40(m, 2 \mathrm{H}), 4.88(\mathrm{ABq}$, $2 \mathrm{H}, J=16.05 \mathrm{~Hz}), 6.80(d, 1 \mathrm{H}, J=7.65 \mathrm{~Hz}), 6.91(t$, $1 \mathrm{H}, J=7.65 \mathrm{~Hz}), 7.18(t, 1 \mathrm{H}, J=7.65 \mathrm{~Hz}), 7.26(t$, $1 \mathrm{H}, J=6.85 \mathrm{~Hz}), 7.32(t, 2 \mathrm{H}, J=7.65 \mathrm{~Hz}), 7.38(d$, $1 \mathrm{H}, J=7.65 \mathrm{~Hz}), 7.52(d, 2 \mathrm{H}, J=7.65 \mathrm{~Hz}), 7.96(m$, $3 \mathrm{H}), 8 \cdot 15$ (br s, 2H, $\mathrm{NH}_{2}, \mathrm{D}_{2} \mathrm{O}$ exchangeable), $8 \cdot 28$ $(d, \quad 1 \mathrm{H}, \quad J=9.15 \mathrm{~Hz}) .{ }^{13} \mathrm{C}$ NMR (DMSO- $d_{6}$, $125 \mathrm{MHz}): \delta 14 \cdot 3,43 \cdot 8,56 \cdot 6,59 \cdot 2,70 \cdot 2,109 \cdot 3$, $123 \cdot 1,124 \cdot 1,127 \cdot 6,127 \cdot 8,127 \cdot 9,128 \cdot 1,128 \cdot 9$, $129 \cdot 2,130 \cdot 0,134 \cdot 8,135 \cdot 6,136 \cdot 6,144 \cdot 4,152 \cdot 8$, 157.1, 163.7, 172.3. MS $(\mathrm{m} / \mathrm{z}): 494\left(\mathbf{M}^{+}\right)$. Anal. Calcd. for $\mathrm{C}_{28} \mathrm{H}_{22} \mathrm{~N}_{4} \mathrm{O}_{5}: \mathrm{C}, 68.01 ; \mathrm{H}, 4.48 ; \mathrm{N}$, $11.33 \%$. Found: C, $67.95 ; \mathrm{H}, 4.42 ; \mathrm{N}, 11.28 \%$.

$2.2 \mathrm{~h}$ 3-Amino-1'-allyl-5, 10-dioxo spiro[(3 H)-indol3',1-5,10-dihydro-1(H)-pyrazolo(1,2-b)phthalazin](1 H)-2'-one-2-carbonitrile (4h) (table 1 , entry 8): pale yellow solid. m.p.: $276-277^{\circ} \mathrm{C} . v_{\max }(\mathrm{KBr})$ : $3382,3261,2200,1712,1690,1667,1612,1561$, $1469,1437,1376,1285,1255,1170,698 \mathrm{~cm}^{-1} .{ }^{1} \mathrm{H}$ NMR (DMSO- $\left.d_{6}, 500 \mathrm{MHz}\right): \delta 4.34(\mathrm{ABq}, 2 \mathrm{H}$, $J=13.0 \mathrm{~Hz}), 5 \cdot 15(d, 1 \mathrm{H}, J=10 \cdot 7 \mathrm{~Hz}), 5 \cdot 31(d, 1 \mathrm{H}$, $J=16.8 \mathrm{~Hz}), 5.82(m, 1 \mathrm{H}), 7.00(d, 1 \mathrm{H}, J=7.6 \mathrm{~Hz})$, $7.06(t, 1 \mathrm{H}, J=7.65 \mathrm{~Hz}), 7.33(t, 1 \mathrm{H}, J=8.4 \mathrm{~Hz})$, $7.53(d, 1 \mathrm{H}, J=7.65 \mathrm{~Hz}), 7.95(m, 3 \mathrm{H}), 8.26(d, 1 \mathrm{H}$, $J=6.85 \mathrm{~Hz}), 8.37\left(b r s, 2 \mathrm{H}, \mathrm{NH}_{2}, \mathrm{D}_{2} \mathrm{O}\right.$ exchangeable). ${ }^{13} \mathrm{C}$ NMR (DMSO- $d_{6}, 125 \mathrm{MHz}$ ): $\delta 42 \cdot 6,60 \cdot 3$, $70 \cdot 0,110 \cdot 3,114 \cdot 9,117 \cdot 0,123 \cdot 8,125 \cdot 0,125 \cdot 3$, $127 \cdot 6,128 \cdot 1,128 \cdot 2,129 \cdot 2,131 \cdot 0,131 \cdot 6,135 \cdot 0$, $135 \cdot 6,143 \cdot 1,152 \cdot 3,153 \cdot 1,156 \cdot 9,171 \cdot 4$. MS $(\mathrm{m} / z)$ : $397\left(\mathrm{M}^{+}\right)$. Anal. Calcd. for $\mathrm{C}_{22} \mathrm{H}_{15} \mathrm{~N}_{5} \mathrm{O}_{3}: \mathrm{C}, 66.49 ; \mathrm{H}$, $3.80 ; \mathrm{N}, 17.62 \%$. Found: $\mathrm{C}, 66.57 ; \mathrm{H}, 3.75 ; \mathrm{N}$, $17.58 \%$. 
2.2i Ethyl 3-amino-1'-allyl-5,10-dioxo spiro[( $3 \mathrm{H})$ indol-3,'1-5,10-dihydro-1(H)-pyrazolo(1,2-b)phthalazin]-(1 H)-2'-one-2-carboxylate (4i) (table 1, entry 9): pale yellow solid. m.p.: $254-256^{\circ} \mathrm{C} . v_{\max }(\mathrm{KBr})$ : $3385,3252,1735,1708,1668,1608,1570,1455$, $1423,1376,1285,1255,1178,692 \mathrm{~cm}^{-1} .{ }^{1} \mathrm{H}$ NMR (DMSO- $\left.d_{6}, 500 \mathrm{MHz}\right): \delta 0.80(s, 3 \mathrm{H}), 3.79(q, 2 \mathrm{H}$, $J=6.9 \mathrm{~Hz}), 4.27(\mathrm{ABq}, 2 \mathrm{H}, J=17.55 \mathrm{~Hz}), 5.18(d$, $1 \mathrm{H}, J=9.95 \mathrm{~Hz}), 5.48(d, 1 \mathrm{H}, J=17.55 \mathrm{~Hz}), 5.82$ $(m, 1 \mathrm{H}), 6.91(t, 1 \mathrm{H}, J=7.6 \mathrm{~Hz}), 6.94(d, 1 \mathrm{H}$, $J=7.65 \mathrm{~Hz}), 7.25(t, 1 \mathrm{H}, J=7.65 \mathrm{~Hz}), 7.35(d, 1 \mathrm{H}$, $J=6.85 \mathrm{~Hz}), 7.86\left(b r s, 2 \mathrm{H}, \mathrm{NH}_{2}, \mathrm{D}_{2} \mathrm{O}\right.$ exchangeable $), 7.95(t, 2 \mathrm{H}, J=7.65 \mathrm{~Hz}), 8.02(d, 1 \mathrm{H}$, $J=9.15 \mathrm{~Hz}), 8.27(d, 1 \mathrm{H}, J=7.9 \mathrm{~Hz}) \cdot{ }^{13} \mathrm{C}$ NMR (DMSO- $\left.d_{6}, 125 \mathrm{MHz}\right): \delta 14.4,43 \cdot 8,59 \cdot 4,70 \cdot 5$, $79 \cdot 7,111 \cdot 5,117 \cdot 9,122 \cdot 9,124 \cdot 2,125 \cdot 3,127 \cdot 1$, $127 \cdot 3,127 \cdot 9,128 \cdot 6,130 \cdot 1,132 \cdot 2,134 \cdot 8,135 \cdot 6$, $152 \cdot 8,153 \cdot 2,157 \cdot 1,162 \cdot 8,171 \cdot 8$. MS $(\mathrm{m} / \mathrm{z}): 444$ $\left(\mathrm{M}^{+}\right)$. Anal. Calcd. for $\mathrm{C}_{24} \mathrm{H}_{20} \mathrm{~N}_{4} \mathrm{O}_{5}: \mathrm{C}, 64.86 ; \mathrm{H}$, $4.54 ; \mathrm{N}, 12.61 \%$. Found: C, 64.75; H, 4.51; N, $12.55 \%$

$2.2 \mathrm{j}$ 3-Amino-5'-nitro-5,10-dioxo spiro[(3 H)-indol3, 1-5, 10-dihydro-1 (H)-pyrazolo (1,2-b)phthalazin](1 H)-2'-one-2-carboxylate (4j) (table 1 , entry 10): Yellow solid. m.p.: $280-282^{\circ} \mathrm{C} . v_{\max }(\mathrm{KBr}): 3378$, $3255,2208,1710,1692,1667,1608,1552,1469$, $1437,1376,1285,1255,1165,698 \mathrm{~cm}^{-1} .{ }^{1} \mathrm{H}$ NMR (DMSO- $\left.d_{6}, 500 \mathrm{MHz}\right): \delta 7 \cdot 12(d, 1 \mathrm{H}, J=8.4 \mathrm{~Hz})$, $7.96(m, 3 \mathrm{H}), 8.24(m, 2 \mathrm{H}), 8.44\left(\right.$ br s $, 2 \mathrm{H}, \mathrm{NH}_{2}$, $\mathrm{D}_{2} \mathrm{O}$ exchangeable), $8.60(d, 1 \mathrm{H}, J=7.65 \mathrm{~Hz}), 11.65$ $\left(s, 1 \mathrm{H}, \mathrm{NH}, \mathrm{D}_{2} \mathrm{O}\right.$ exchangeable). ${ }^{13} \mathrm{C}$ NMR (DMSO$\left.d_{6}, 125 \mathrm{MHz}\right): \delta 64 \cdot 0,69.6,111 \cdot 2,114.8,121 \cdot 6$, $127 \cdot 5,128 \cdot 0,128 \cdot 1,129 \cdot 5,135 \cdot 0,135 \cdot 6,143 \cdot 6$, $148 \cdot 7,152 \cdot 7,153 \cdot 1,157 \cdot 0,173 \cdot 7$. MS $(\mathrm{m} / z): 402$ $\left(\mathrm{M}^{+}\right)$. Anal. Calcd. for $\mathrm{C}_{19} \mathrm{H}_{10} \mathrm{~N}_{6} \mathrm{O}_{5}: \mathrm{C}, 56.72 ; \mathrm{H}$, $2.51 ; \mathrm{N}, 20.89 \%$. Found: C, 56.80; H, 2.47; N, $20.82 \%$.

\section{Results and discussion}

As part of our endeavour to discover new spirooxindoles of biocidal interest, and guided by the observation that the presence of two or more different heterocyclic moieties in a single molecule often enhances the biocidal profile remarkably, we investigated a three-component reaction of isatin with malononitrile and phthalhydrazide, in order to synthesize a new class of spirooxindoles with fused phthalazines. To the best of our knowledge, there have been no reports on the synthesis of pyra- zolophthalazinyl spiro-3'-oxindoles. Heterocyclic fused phthalazines have been found effective for the inhibition of p38 MAP kinase, ${ }^{6}$ selective binding of GABA receptor, ${ }^{7}$ antianxiety drug, ${ }^{8}$ antitumour agent, ${ }^{9}$ high affinity ligands to the $\alpha_{2} \delta-1$ subunit of calcium channel. ${ }^{10}$

Recently, L-proline has been effectively used as a versatile organocatalyst in various organic transformations. ${ }^{11}$ In an extension of our continuing efforts on the application of malononitrile based multicomponent reactions in heterocyclic synthesis and in the synthesis of spirooxindoles, ${ }^{12}$ we report here a simple and efficient method for the synthesis of pyrazolophthalazinyl spiro-3'-oxindoles, through the three-component condensation of isatin, malononitrile and phthalhydrazide using L-proline as a catalyst (scheme 1).

Initially, a model one-pot three-component reaction of isatin $1 \mathbf{a}(1 \mathrm{mmol})$, malononitrile $\mathbf{2 a}(1 \mathrm{mmol})$ and phthalhydrazide 3 (1 mmol) was investigated. Various catalysts, including $\mathrm{InCl}_{3}, \mathrm{CAN}, \mathrm{NH}_{2} \mathrm{SO}_{3} \mathrm{H}$, $\mathrm{SnCl}_{2} \cdot 2 \mathrm{H}_{2} \mathrm{O}$, L-proline, $\mathrm{K}_{2} \mathrm{CO}_{3}$ and basic alumina were screened in our model reaction (table 2). The best overall yield (90\%) was obtained with L-proline in ethanol. Optimum results were obtained using $20 \mathrm{~mol} \%$ of L-proline.

Table 1 summarizes our results on the one-pot reaction of various isatin derivatives and malononitrile/ ethyl cyanoacetate with phthalhydrazide (scheme 2). All the completed reactions afforded the corresponding pyrazolophthalazinyl spiro-3'-oxindoles in good yields.

The structures of compounds $\mathbf{4 a} \mathbf{a}-\mathbf{j}$ were confirmed by IR, ${ }^{1} \mathrm{H}$ and ${ }^{13} \mathrm{C}$ NMR spectroscopy, mass spectrometry and elemental analysis. The mass spectrum of $4 \mathbf{a}$ displayed the molecular ion $\left(\mathbf{M}^{+}\right)$peak at $\mathrm{m} / \mathrm{z}$ 357. The ${ }^{1} \mathrm{H}$ NMR spectrum of $4 \mathrm{a}$ exhibited a broad singlet at $\delta 8.32\left(\mathrm{D}_{2} \mathrm{O}\right.$ exchangeable $)$ due to $-\mathrm{NH}_{2}$, a

Table 2. One-pot, three-component synthesis of $\mathbf{4 a}$ using various catalysts.

\begin{tabular}{llcc}
\hline Entry & Catalyst & Time (h) & Yield $^{\text {a }}(\%)$ \\
\hline 1 & $\mathrm{No} \mathrm{cat.}$ & 24 & - \\
2 & $\mathrm{InCl}_{3}$ & 6 & 45 \\
3 & $\mathrm{CAN}_{2}$ & 10 & - \\
4 & $\mathrm{NH}_{2} \mathrm{SO}_{3} \mathrm{H}$ & 9 & 10 \\
5 & $\mathrm{SnCl}_{2} \cdot 2 \mathrm{H}_{2} \mathrm{O}$ & 8 & 34 \\
6 & $\mathrm{~L}_{-} \mathrm{proline}_{2}$ & 2 & 90 \\
7 & $\mathrm{~K}_{2} \mathrm{CO}_{3}$ & 6 & 30 \\
8 & Basic alumina & 6 & 20 \\
\hline
\end{tabular}


<smiles>O=C1Nc2ccccc2C1=O</smiles>

$+<_{\mathrm{CN}}^{\mathrm{CN}}+$<smiles>O=c1[nH][nH]c(=O)c2ccccc12</smiles><smiles>[TeH4]</smiles>
ethanol/reflux<smiles></smiles>

Scheme 1.<smiles>O=C1C(=[OH+])N(P)c2ccc(Br)cc21</smiles><smiles>O=C1C(=O)N(P)c2ccc(Br)cc21</smiles><smiles>[X]C1CCCCC1</smiles>

2<smiles>O=c1[nH][nH]c(=O)c2ccccc12</smiles><smiles>[X]C1=C(N)n2c(=O)c3ccccc3c(=O)n2C12C(=O)N([R])c1ccc([R])cc12</smiles>

Scheme 2.<smiles>[X]C(C#N)=C1C(=O)N(P)c2ccc([R])cc21</smiles>

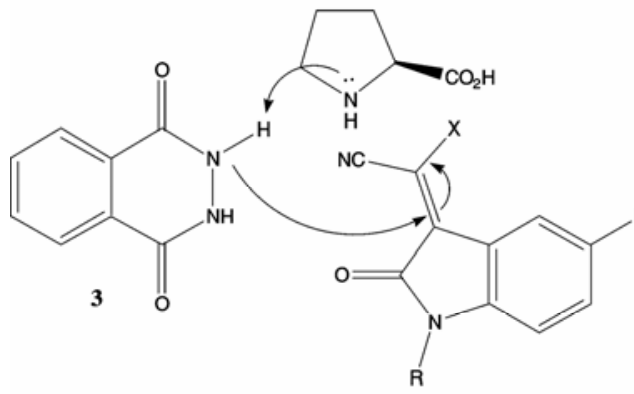<smiles>[R]c1ccc2c(c1)C(C([X])C#N)(n1[nH]c(=O)c3ccccc3c1=O)C(=O)N2[R]</smiles><smiles>[X]C1=C(N)n2c(=O)c3ccccc3c(=O)n2C12C(=O)N([R])c1ccc([R])cc12</smiles>

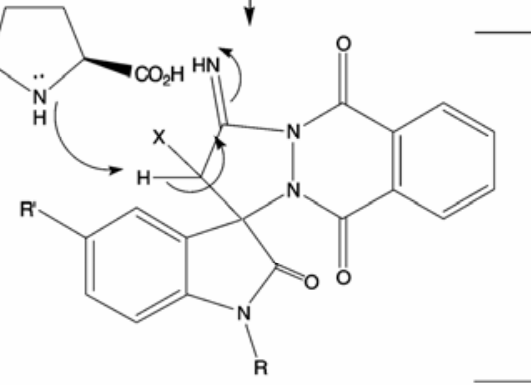

Scheme 3.

singlet at $\delta 10.92\left(\mathrm{D}_{2} \mathrm{O}\right.$ exchangeable $)$ due to $-\mathrm{NH}$ isatin and aromatic protons in the range $\delta 6 \cdot 90-8 \cdot 25$. Resonances at $\delta 70.3$ (spiro carbon), $\delta 153 \cdot 1$ and $156.9(-\mathrm{C}=\mathrm{O}$ groups of phthalazine) and $\delta 173.0$ (isatin $-\mathrm{C}=\mathrm{O}$ group) were observed in the ${ }^{13} \mathrm{C}$ NMR spectrum. Furthermore, the structure of $\mathbf{4 b}$ was established by X-ray crystallographic analysis ${ }^{13}$ (figure 2). 


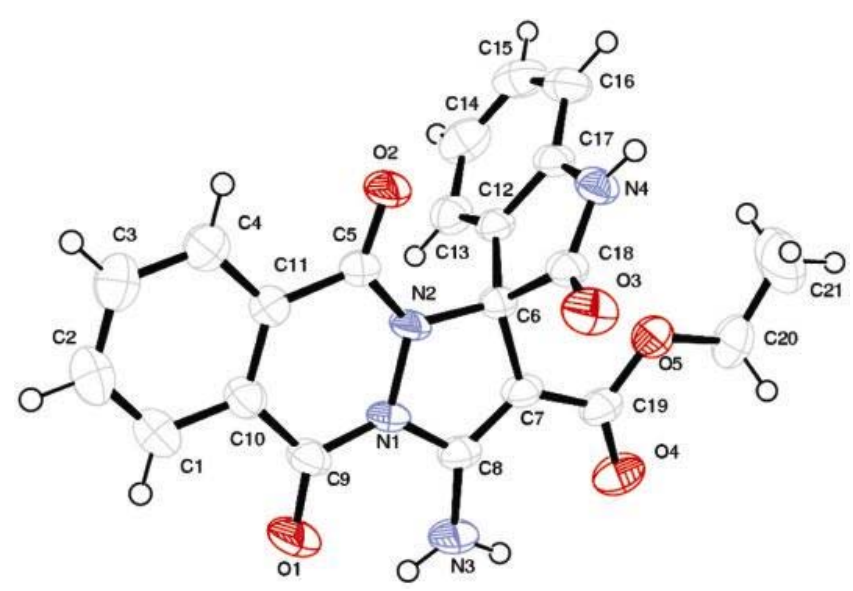

Figure 2. ORTEP diagram of compound $\mathbf{4 b}$.

A possible mechanism for the formation of $4 \mathbf{a}-\mathbf{j}$ is proposed in scheme 3 . The process represents a typical cascade reaction in which the isatin 1 first condenses with malononitrile/ethyl cyanoacetate $\mathbf{2}$ to afford isatylidene malononitrile derivative 5. This step can be regarded as a fast Knoevenagel addition. Then, the subsequent Michael type addition of the phthalhydrazide $\mathbf{3}$ to $\mathbf{5}$ followed by cyclization affords the corresponding product $\mathbf{4 a - j}$.

\section{Conclusion}

In conclusion, we have developed a simple and clean procedure for the synthesis of new pyrazolophthalazinyl spiro-3'-oxindoles starting from commercially available starting materials. The use of inexpensive and readily available L-proline has made this procedure simple, convenient and practical.

\section{Acknowledgement}

One of the authors, G S thanks the Council of Scientific and Industrial Research, New Delhi, India for the research fellowship.

\section{References}

1. (a) Tour B B and Hall D G 2009 Chem. Rev. 109 4439; (b) Ganem B 2009 Acc. Chem. Res. 42 463; (c) Tejedor D and Garcia-Tellado F 2007 Chem. Soc. Rev. 36 484; (d) Ugi I and Heck S 2001 Comb. Chem. High Throughput Screening 4 1; (e) Ugi I, Domling
A and Werner B 2000 J. Heterocycl. Chem. 37 647; (f) Bienayme H, Hulme C, Oddon G and Schmitt P 2000 Chem. Eur. J. 63321

2. (a) Fontaine P, Masson G and Zhu J 2009 Org. Lett. 11 1555; (b) Radi M, Bernardo V, Bechi B, Castagnolo D, Pagano M and Botta M 2009 Tetrahedron Lett. 50 6572; (c) Teimouri M B, Abbasi T and Mivehchi H 2008 Tetrahedron 64 10425; (d) Santra S and Andreana P R 2007 Org. Lett. 95035

3. (a) Castaldi M P, Troast D M and Porco Jr J A 2009 Org. Lett. 11 3362; (b) Volk B, Barkoczy J, Hegedus E, Udvari S, Gacsalyi I, Mezei T, Pallagi K, Kompagne H, Levay G, Egyed A, Harsing Jr L G, Spedding M and Simig G 2008 J. Med. Chem. 51 2522; (c) Hamashima Y, Suzuki T, Takano H, Shimura Y and Sodeoka M $2005 \mathrm{~J}$. Am. Chem. Soc. 127 10164; (d) Marti C and Carreira E M 2003 Eur. J. Org. Chem. 632209

4. (a) Khafagy M M, El-Wahas A H F A, Eid F A and El-Agrody A M 2002 Farmaco 57 715; (b) Sebahar P $\mathrm{R}$ and Williams R M $2000 \mathrm{~J}$. Am. Chem. Soc. 122 5666

5. Kang T H, Matsumoto K, Murakami Y, Takayama H, Kitajima M, Aimi N and Watanabe H 2002 Eur. J. Pharmacol. 44439

6. Mavel S, Thery I and Gueiffier A 2002 Arch. Pharm. Med. Chem. 3357

7. (a) Atack J R 2010 Pharmacology and Therapeutics 125 11; (b) Carling R W, Moore K W, Street L J, Wild D, Isted C, Leeson P D, Thomas S, O'Connor D, McKernan R M, Quirk K, Cook S M, Atack J R, Wafford K A Thompson S A, Dawson G R, Ferris P and Castro J L $2004 \mathrm{~J}$. Med. Chem. 471807

8. Imamura $\mathrm{Y}$, Noda A, Imamura $\mathrm{T}$, Ono $\mathrm{Y}$, Okawara $\mathrm{T}$ and Noda H 2003 Life Sci. 7429

9. Kim J S, Rhee H-K, Park H J, Lee S K, Lee C-O and Choo H-Y P 2008 Bioorg. Med. Chem. 164545

10. Lebsack A D, Gunzner J, Wang B, Pracitto R, Schaffhauser H, Santini A, Aiyar J, Bezverkov R, Munoz B, Liu W and Venkatraman S 2004 Bioorg. Med. Chem. Lett. 142463

11. (a) Diao X, Wang Y, Jiang Y and Ma D 2009 J. Org. Chem. 74 7974; (b) Zhou Y and Shan Z 2007 J. Org. Chem. 72 686; (c) Dodda R and Zhao C-G 2006 Synthesis 19 3238; (d) Wang Y, Shang Z-C, Wu T-X, Fan J-C and Chen X $2006 \mathrm{~J}$. Mol. Catal. A: Chem. 253 212; (e) Shen Z, Li B, Wang L and Zhang Y 2005 Tetrahedron Lett. 468785

12. (a) Shanthi G, Subbulakshmi G and Perumal P T 2007 Tetrahedron 63 2057; (b) Shanthi G and Perumal P T 2007 Tetrahedron Lett. 48 6785; (c) Shanthi G and Perumal P T 2008 Tetrahedron Lett. 49 7139; (d) Shanthi G and Perumal P T 2008 Synlett. 2791; (e) Jayashree P, Shanthi G and Perumal P T 2009 Synlett. 917

13. Crystal structure of compound $\mathbf{4 b}$ was deposited at the Cambridge Crystallographic Data Center and allocated the reference no. CCDC 656685 